http://dx.doi.org/10.22319/rmcp.v9i2.4484

Nota de Investigación

\title{
Evaluación morfológica de gallinas de traspatio mexicanas (Gallus gallus domesticus)
}

\section{Morphological evaluation Mexican backyard chickens \\ (Gallus gallus domesticus)}

Vicente Eliezer Vega Murillo ${ }^{\mathrm{a}}$

Sergio I. Román Ponce ${ }^{b^{*}}$

Marina Durán Aguilar ${ }^{\mathrm{c}}$

Alejandra Vélez Izquierdo ${ }^{\mathrm{b}}$

Eduardo Cabrera Torres ${ }^{\mathrm{d}}$

Antonio Cantú Covarrubias ${ }^{\mathrm{e}}$

Lino De la Cruz Colín ${ }^{\mathrm{f}}$

Jorge Alonso Maldonado Jaquez ${ }^{\mathrm{g}}$

Guillermo Martínez Velázquez ${ }^{\mathrm{h}}$

Ángel Ríos Utrera ${ }^{\mathrm{a}}$,

Alessandro Bagnato ${ }^{\mathrm{i}}$

Maria Giuseppina Strillacci ${ }^{i}$

Moisés Montaño Bermúdez ${ }^{\mathrm{b}}$

Felipe J. Ruiz López ${ }^{\mathrm{b}}$

a Campo Exp. La Posta, INIFAP, km 22.5 carretera federal Veracruz-Cordoba, Tel: 01 (229) 262-2222. Paso del Toro, 94277. Veracruz, México.

b Centro Nacional de Investigación Disciplinaria en Fisiología y Mejoramiento Animal, INIFAP. Ajuchitlán, Querétaro. México. 
${ }^{\mathrm{c}}$ Facultad de Estudios Superiores Cuautitlán; UNAM. Estado de México. México.

${ }^{\mathrm{d}}$ Campo Exp. Chetumal; CIRSE, INIFAP. Chetumal, Quintana Roo. México.

${ }^{\text {e }}$ Sitio Experimental Aldama, CIRNE, INIFAP. Aldama, Tamaulipas. México.

${ }^{\mathrm{f}}$ Campo Exp. Valle de México, CIRCE, INIFAP. Texcoco, Estado de México.

${ }^{g}$ Campo Exp. La Laguna, CIRNOC, INIFAP. Matamoros, Coahuila. México.

${ }^{\text {h }}$ Campo Exp. Santiago Ixcuintla, CIRPAC, INIFAP. Santiago Ixcuintla, Nayarit. México.

${ }^{\mathrm{i}}$ Università degli Studi di Milano. Dipartimento di Scienze e Tecnologie Veterinarie per la Sicurezza Alimentare. Milán, Italia.

Autor de correspondencia: roman.sergio@inifap.gob.mx

\section{- Resumen:}

El objetivo fue evaluar algunas características morfológicas de gallinas de traspatio ( $\mathrm{n}=$ 255) provenientes de 65 unidades rurales de producción, localizadas en 52 municipios de 18 estados de la República Mexicana. El modelo estadístico incluyó los efectos de sexo, estado y municipio anidado en estado. El efecto de sexo fue importante para todas las variables analizadas $(P<0.0001)$, excepto para robustez $(P=0.33)$. El efecto de estado fue importante $(P<0.0001)$ para todas las variables analizadas, mientras que municipio solo influyó significativamente a envergadura $(P<0.0001)$, peso corporal $(P=0.0271)$ y solidez $(P=0.0267)$. Los machos tuvieron mayor $(P<0.0001)$ longitud corporal $(4.85 \mathrm{~cm})$, envergadura $(5.66 \mathrm{~cm})$, circunferencia de la pechuga $(2.94 \mathrm{~cm})$, longitud del tarso $(1.73$ $\mathrm{cm})$, peso corporal $(0.65 \mathrm{~kg})$ y solidez (0.99 puntos porcentuales). El peso corporal aumentó $126 \mathrm{~g}(P<0.01)$ en machos y $61 \mathrm{~g}$ en hembras $(P<0.01)$ por cada centímetro que aumentó la circunferencia de la pechuga. El peso corporal mostró estar moderadamente correlacionado con longitud del cuerpo $(r=0.65)$, envergadura $(r=0.49)$ y longitud del tarso $(r=0.67)$, y altamente correlacionada con circunferencia de la pechuga $(r=0.76)$ en machos. La gallina de traspatio en México presentó una diferenciación morfológica importante entre machos y hembras para las características estudiadas, excepto para robustez, en la cual no se detectaron diferencias.

- Palabras Clave: Gallinas de traspatio, Caracterización, Morfología.

\section{- Abstract:}

The objective was to evaluate some morphological characteristics of backyard chickens ( $\mathrm{n}=$ 255) coming from 65 rural production units located in 52 municipalities of 18 States of the 
Mexican Republic. The statistical model included sex, state, and municipality within state. Sex affected all the response variables $(P<0.0001)$, except massiveness index $(P=0.3369)$. State affected all the response variables $(P<0.0001)$ and municipality was only important for wingspan $(P<0.0001)$, body weight $(P<0.0271)$ and stockiness index $(P<0.0267)$. Male chickens had greater $(P<0.0001)$ body length $(4.85 \mathrm{~cm}$ more $)$, wingspan $(5.66 \mathrm{~cm}$ more $)$, breast circumference $(2.94 \mathrm{~cm}$ more $)$, shank length $(1.73 \mathrm{~cm}$ more $)$, body weight $(0.65 \mathrm{~kg}$ more) and massiveness index ( 0.99 percentage points) than female chickens. Body weight increased $126 \mathrm{~g}(P<0.01)$ in males and $61 \mathrm{~g}$ in females $(P<0.01)$ for each centimeter increment in breast circumference. Body weight showed to be moderately correlated $(P<0.01)$ with body length $(\mathrm{r}=0.65)$, wing span $(\mathrm{r}=0.49)$, length of the shank $(\mathrm{r}=0.67)$ and highly correlated with breast circumference ( $\mathrm{r}=0.76$ in males). The Mexican backyard chicken presented significant morphological differences between males and females for traits studied, except for massiveness index.

- Key words: Backyard chickens, Characterization, Morphology.

Recibido 9/05/2017.

Aceptado 30/09/2017.

La avicultura de traspatio es el aprovechamiento de animales, como gallinas, guajolotes, patos y otras aves en el patio de la casa o alrededor de la misma, siendo su característica principal que es una avicultura de pequeña escala, realizada -en la mayoría de los casos- en áreas rurales, suburbanas y zonas marginadas ${ }^{(1)}$. La productividad de las gallinas de traspatio es menor que las de las razas mejoradas o las cruzas utilizadas por la avicultura industrial, pero sus costos de producción son mínimos ${ }^{(2)}$. La finalidad principal de la producción es el autoconsumo familiar y venta de excedentes. Además, proporciona proteína de origen animal, mejora la economía con la venta de huevo o carne, y complementa a la avicultura comercial para un mercado que demanda productos diferenciados; por esto, se convierte en una escala económica difícil de medir ${ }^{(3)}$.

Diversos estudios han determinado la diversidad morfológica y genética de la gallina de traspatio mexicana proveniente de algunas regiones de nuestro país ${ }^{(4-8)}$. Sin embargo, es necesaria la evaluación de gallinas provenientes de un mayor número de regiones agroecológicas y sistemas de producción. Con base en lo anterior, el objetivo del presente estudio fue evaluar características morfológicas cuantitativas, de gallinas de traspatio 
provenientes de comunidades rurales de la República Mexicana, así como estimar las correlaciones fenotípicas entre las variables morfológicas cuantitativas.

Se analizó la información morfológica de 255 gallinas de traspatio resultante de un muestreo por oportunidad, que incluyó 65 unidades rurales de producción ubicadas en 52 municipios de 18 Estados de la República Mexicana: Aguascalientes, Baja California Norte, Baja California, Chihuahua, Coahuila, Colima, Distrito Federal, Durango, Estado de México, Guanajuato, Guerrero, Jalisco, Morelos, Nayarit, Nuevo León, Oaxaca, Tamaulipas y Veracruz. Los municipios muestreados se presentan en el Cuadro 1. La información se recolectó de 2013 a 2014 a partir de gallinas de ambos sexos (78 machos y 179 hembras), tanto jóvenes como adultos.

Cuadro 1: Municipios muestreados enlistados por estado

\begin{tabular}{ll}
\hline Estado & \\
\hline Aguascalientes & Calvillo, Cosío \\
Baja California Norte & Ensenada \\
Baja California Sur & Comundú, La Paz, Los Cabos \\
Chihuahua & Casas Grandes, Janos, Nuevo Casas Grandes \\
Coahuila & Matamoros, Torreón, Zaragoza \\
Colima & Cómala, Cuauhtémoc, Manzanillo, Minatitlán \\
Ciudad de México & Milpa Alta \\
Durango & Cuencamé, Durango, Guadalupe Victoria \\
Estado de México & Almoloya de Juárez, Ixtlahuaca, Jocotitlán, San Felipe Del Progreso, \\
Guanajuato & Santa Maria Ajoloapan \\
Guerrero & Abasolo, Comonfort, Huanímaro, Salamanca, Valle de Santiago \\
Jalisco & Ometepec \\
Morelos & Cabo Corrientes, Cuautitilán de García Barragán, Villa Purificación \\
Nayarit & Miacatlan, Temixco, Tlalquiltenango \\
Nuevo León & Compostela, Del Nayar, Ruiz, Santiago Ixcuintla \\
Oaxaca & General Bravo, Linares \\
Tamaulipas & Ciudad Ixtepec, Ejutla de Crespo, Loma Bonita, Matías Romero, \\
Veracruz & San Vicente Coatlan \\
\hline & Aldama, Altamira, Nuevo Laredo \\
Medellín
\end{tabular}


Las variables evaluadas fueron: longitud del cuerpo, envergadura, circunferencia de la pechuga, longitud del tarso, peso vivo corporal, robustez y solidez. Las mediciones de las variables cuantitativas se realizaron siguiendo los Lineamientos para la Producción Animal y la Salud de la Organización de las Naciones Unidas para la Alimentación y la Agricultura $^{(9)}$. El peso de las gallinas se obtuvo con un dinamómetro colgante con capacidad para $10 \mathrm{~kg}$ (marca PEXA; modelo ECO-DIN 10; precisión $\pm 25 \mathrm{~g}$ ), mientras que longitud del cuerpo, envergadura, circunferencia de la pechuga y longitud del tarso se midieron con una cinta métrica flexible de plástico (marca FIBER-GLASS). Las siete variables se definieron de la siguiente manera:

Longitud del cuerpo. Se midió como la distancia en centímetros que existe entre la base del pico y el extremo caudal, a la altura de la glándula uropígea, sin considerar las plumas de la cola, teniendo el pescuezo del animal extendido.

Envergadura. Se midió como la distancia en centímetros, de la falange terminal de un ala a la falange terminal de la otra ala (sin incluir las plumas), manteniendo las alas del ave completamente extendidas.

Circunferencia de la pechuga. Se midió en centímetros, a nivel de la punta de la quilla, pasando la cinta métrica por la parte posterior de la inserción de las alas.

Longitud del tarso. Correspondió al largo del tarso-metatarso y se definió como la distancia de la articulación intertarsiana a la articulación metatarsofalángica, en centímetros.

Peso corporal. Correspondió al peso del animal vivo en kilogramos, medido con una báscula en el momento en que se realizó la visita a la unidad de producción.

Robustez. Se definió como: circunferencia de la pechuga/longitud corporal x 100.

Solidez. Se definió como peso corporal/longitud corporal x 100 .

Se realizó un análisis de varianza para cada característica con el procedimiento GLM de $\mathrm{SAS}^{(10)}$. En todos los casos, el modelo estadístico incluyó los efectos de sexo, estado y municipio anidado dentro de estado. Matemáticamente, el modelo estadístico utilizado fue el siguiente:

$$
\mathrm{y}_{\mathrm{ijkl}}=\mu+\alpha_{\mathrm{i}}+\beta_{\mathrm{j}}+\gamma_{\mathrm{k}(\mathrm{j})}+\varepsilon_{\mathrm{ijk} \mathrm{l}},
$$

\section{Donde:}

$\mathbf{y}_{\mathrm{ij} \mathbf{j} \mathrm{l}=}$ es la i-ésima observación de la variable de respuesta (longitud del cuerpo, envergadura, circunferencia de la pechuga, longitud del tarso, peso vivo corporal, robustez o solidez),

$\boldsymbol{\mu}=$ es la media general, $\alpha_{i}=$ es el efecto fijo del i-ésimo sexo (i=1,2), 
$\boldsymbol{\beta}_{\mathbf{j}}=$ es el efecto fijo del $\mathbf{j}$-ésimo estado $(\mathrm{j}=1, \ldots, 18)$,

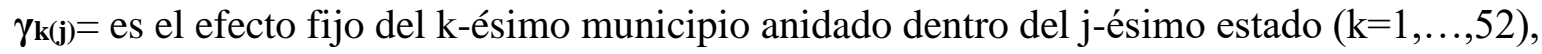

$\varepsilon_{\mathrm{ijk} k}=$ es el l-ésimo error aleatorio,

$\mathrm{y}_{\mathrm{ijkl}} \sim N\left(\mu, \sigma^{2}\right)$.

Las diferencias entre las medias de machos y hembras se estimaron con la opción PDIFF del procedimiento GLM. Adicionalmente, con el procedimiento CORR de $\operatorname{SAS}^{(10)}$ se estimaron los coeficientes de correlación de Pearson para las variables longitud del cuerpo, envergadura, circunferencia de la pechuga, longitud del tarso y peso corporal, con el fin de conocer su asociación con el peso corporal. Las correlaciones se estimaron para machos y hembras de manera independiente y para datos conjuntos de machos y hembras. Se estimó el coeficiente de regresión lineal del peso corporal sobre la circunferencia de la pechuga del ave, utilizando el procedimiento REG de $S A S^{(10)}$. Este análisis también se hizo para machos y hembras de manera independiente, y para datos conjuntos de machos y hembras. Las frecuencias de las categorías observadas en campo, correspondientes a cada una de las características cualitativas estudiadas, se calcularon con el procedimiento FREQ de SAS ${ }^{(10)}$.

En el Cuadro 2 se muestran las estadísticas descriptivas de las variables morfológicas cuantitativas de las gallinas de traspatio. Las gallinas muestreadas pesaron en promedio 1.41 y $2.11 \mathrm{~kg}$ para hembras y machos, respectivamente Estos valores son menores a los obtenidos en el estado de Chiapas $^{(4)}$ de 1.90 y $2.30 \mathrm{~kg}$, y similares a los obtenidos en el estado Puebla ${ }^{(11)}$ de 1.60 y $2.12 \mathrm{~kg}$ para para machos y hembras, respectivamente. El mayor coeficiente de variación (CV) fue para peso corporal, el cual fue al menos dos veces mayor que el de las otras características analizadas, y los menores fueron para longitud del cuerpo y circunferencia de la pechuga. En un estudio de diferenciación morfométrica y evaluación de la función de ecotipos de gallinas nativas en Nigeria ${ }^{(12)}$, se observaron, en promedio, menores CV para peso corporal, envergadura y longitud del cuerpo en machos y hembras ( 18.29 y $15.99 \% ; 8.42$ y $10.95 \%$ y 8.32 y $7.33 \%$, respectivamente) que los encontrados en este estudio. 
Cuadro 2: Estadísticas descriptivas de variables morfológicas cuantitativas de gallinas de traspatio, por sexo

\begin{tabular}{|c|c|c|c|c|c|c|c|}
\hline Variable & Sexo & $\mathbf{N}$ & Media & DE & $\begin{array}{c}\text { Valor } \\
\text { mínimo }\end{array}$ & $\begin{array}{c}\text { Valor } \\
\text { máximo }\end{array}$ & $\begin{array}{l}\mathrm{CV} \\
(\%)\end{array}$ \\
\hline \multirow{3}{*}{$\begin{array}{l}\text { Circunferencia de la } \\
\text { pechuga, } \mathrm{cm}\end{array}$} & $\mathrm{M}$ & 74 & 31.41 & 5.02 & 19.00 & 42.00 & 16.0 \\
\hline & $\mathrm{H}$ & 170 & 28.85 & 4.30 & 17.00 & 41.00 & 14.9 \\
\hline & Promedio & 244 & 30.13 & 4.66 & 18.00 & 41.50 & 15.8 \\
\hline \multirow{3}{*}{ Envergadura, cm } & $\mathrm{M}$ & 78 & 44.59 & 8.93 & 21.00 & 65.00 & 20.0 \\
\hline & $\mathrm{H}$ & 177 & 39.59 & 8.19 & 22.00 & 64.00 & 20.7 \\
\hline & Promedio & 255 & 42.09 & 8.56 & 21.50 & 64.50 & 21.2 \\
\hline \multirow{3}{*}{$\begin{array}{l}\text { Longitud del tarso, } \\
\mathrm{cm}\end{array}$} & $\mathrm{M}$ & 78 & 9.69 & 1.84 & 5.00 & 15.00 & 19.0 \\
\hline & $\mathrm{H}$ & 179 & 7.79 & 1.21 & 5.00 & 11.00 & 15.5 \\
\hline & Promedio & 257 & 8.74 & 1.53 & 5.00 & 13.00 & 20.0 \\
\hline \multirow{3}{*}{$\begin{array}{l}\text { Longitud del } \\
\text { cuerpo, cm }\end{array}$} & $\bar{M}$ & 77 & 40.92 & 7.73 & 18.00 & 58.00 & 18.9 \\
\hline & $\mathrm{H}$ & 179 & 37.10 & 5.30 & 21.00 & 52.00 & 14.3 \\
\hline & Promedio & 256 & 39.01 & 6.52 & 19.50 & 55.00 & 16.6 \\
\hline \multirow{3}{*}{ Peso corporal, kg } & $\mathrm{M}$ & 77 & 2.11 & 0.85 & 0.25 & 3.80 & 40.1 \\
\hline & $\mathrm{H}$ & 177 & 1.41 & 0.49 & 0.30 & 2.80 & 35.0 \\
\hline & Promedio & 254 & 1.76 & 0.67 & 0.28 & 3.30 & 43.0 \\
\hline \multirow{3}{*}{ Robustez } & $\bar{M}$ & 74 & 77.70 & 13.14 & 54.17 & 133.30 & 16.9 \\
\hline & $\mathrm{H}$ & 170 & 79.04 & 15.32 & 36.96 & 164.00 & 19.4 \\
\hline & Promedio & 244 & 78.37 & 14.23 & 45.57 & 148.65 & 18.7 \\
\hline \multirow{3}{*}{ Solidez } & $\mathrm{M}$ & 77 & 5.05 & 1.70 & 1.39 & 10.42 & 33.7 \\
\hline & $\mathrm{H}$ & 177 & 3.85 & 1.40 & 1.14 & 10.00 & 36.5 \\
\hline & Promedio & 254 & 4.45 & 1.55 & 1.27 & 10.21 & 37.9 \\
\hline
\end{tabular}

$\mathrm{DE}=$ desviación estándar; $\mathrm{M}=$ machos; $\mathrm{H}=$ hembras; $\mathrm{CV}=$ coeficiente de variación.

El efecto de sexo fue importante para todas las variables analizadas $(P<0.001)$, excepto para robustez. El efecto de estado fue importante $(P<0.001)$ para todas las variables analizadas, mientras que municipio sólo influyó significativamente $(P<0.05)$ a envergadura, peso corporal y solidez.

Las medias de cuadrados mínimos y errores estándar de las variables cuantitativas estudiadas para gallinas machos y hembras se presentan en el Cuadro 3. Los machos tuvieron mayor $(P<0.001)$ longitud corporal $(4.85 \mathrm{~cm}$ más $)$, envergadura $(5.66 \mathrm{~cm}$ más $)$, circunferencia de la pechuga (2.94 cm más), longitud del tarso (1.73 cm más), peso corporal (0.65 kg más) y solidez (9.9 puntos porcentuales más) que las hembras. En un estudio de evaluación zoométrica de una población de gallinas de las tierras altas del sureste mexicano ${ }^{(4)}$, se observó que los machos pesaron desde 1,260 g hasta 4,200 g, mientras que 
las hembras de 1,020 hasta 3,050 g (17 \% más pesados los machos que las hembras $(P<0.01)$. De manera similar a este estudio, en gallinas y gallos de la misma edad $(20$ y 32 semanas) de tres localidades en Oaxaca ${ }^{(13)}$, se observaron pesos vivos promedio para machos de 2.75 a $3.00 \mathrm{~kg}$, y para hembras de 1.80 a $2.00 \mathrm{~kg}$ (34\% más pesados los machos que las hembras). En un estudio realizado en la comunidad de La Trinidad Tianguismanalco, municipio de Tecali de Herrera, en el estado de Puebla ${ }^{(11)}$, reportaron pesos corporales de animales adultos de $2.13 \pm 0.51$ y $1.63 \pm 0.32 \mathrm{~kg}$ en machos y hembras, respectivamente, lo que indica una adaptación de las diferentes cruzas de aves que maneja la familia en las condiciones del ambiente prevaleciente.

Cuadro 3: Medias de cuadrados mínimos y errores estándar de variables morfológicas cuantitativas de gallinas de traspatio, por sexo

\begin{tabular}{lccccccc}
\hline Sexo & LC $(\mathbf{c m})$ & ENV $(\mathrm{cm})$ & CP $(\mathbf{c m})$ & LT $(\mathbf{c m})$ & PC $(\mathbf{k g})$ & RO $(\%)$ & SO (\%) \\
\hline Hembras & $35.53 \pm 0.61^{\mathrm{a}}$ & $39.19 \pm 0.69^{\mathrm{a}}$ & $28.70 \pm 0.48^{\mathrm{a}}$ & $7.93 \pm 0.15^{\mathrm{a}}$ & $1.46 \pm 0.06^{\mathrm{a}}$ & $81.91 \pm 1.42$ & $4.17 \pm 0.15^{\mathrm{a}}$ \\
Machos & $40.38 \pm 0.74^{\mathrm{b}}$ & $44.85 \pm 0.83^{\mathrm{b}}$ & $31.64 \pm 0.59^{\mathrm{b}}$ & $9.66 \pm 0.18^{\mathrm{b}}$ & $2.11 \pm 0.08^{\mathrm{b}}$ & $80.22 \pm 1.75$ & $5.16 \pm 0.18^{\mathrm{b}}$ \\
\hline
\end{tabular}

$\mathrm{LC}=$ longitud del cuerpo; $\mathrm{ENV}=$ envergadura; $\mathrm{CP}=$ circunferencia de la pechuga; $\mathrm{LT}=$ longitud del tarso; $\mathrm{PC}=$ peso corporal; $\mathrm{RO}=$ robustez; $\mathrm{SO}=$ solidez.

${ }^{\mathrm{a}, \mathrm{b}}$ Medias por sexo con diferente literal son distintas $(P<0.001)$.

En una caracterización fenotípica de gallinas criollas en Colombia ${ }^{(14)}$, observaron que los machos tuvieron longitudes del tarso mayores que las hembras, similar a lo encontrado en el presente estudio (11.49 vs $8.37 \mathrm{~cm}$ ). De manera similar, en tres localidades en Oaxaca ${ }^{(13)}$, observaron que el largo del tarso en machos de 20 semanas fue de $9.6 \mathrm{~cm}$ en promedio y en gallinas adultas fue en promedio de $9.8 \mathrm{~cm}$.

Las diferencias entre machos y hembras para circunferencia de la pechuga en este estudio son similares a las encontradas en tres comunidades estudiadas en el estado de Oaxaca ${ }^{(13)}$, de $15.6 \%$ de diferencia entre machos y hembras a las 20 y 32 semanas. La superioridad de machos sobre hembras para las características en estudio también ha sido observada en otros países, como España ${ }^{(15,16)}$, Ecuador ${ }^{(17)}$ Nigeria $^{(12)}$, Colombia $^{(18)}$ y Guatemala $^{(19)}$.

Los análisis de regresión mostraron que el coeficiente de regresión del peso corporal sobre la circunferencia de la pechuga fue diferente de cero $(P<0.001)$, tanto en machos como en hembras, así como en los datos agrupados. En machos, el peso corporal aumentó 126 g por cada centímetro que aumentó la circunferencia de la pechuga, mientras que en hembras el peso corporal aumentó $61 \mathrm{~g}$ por cada centímetro que aumentó la circunferencia de la pechuga. El análisis de regresión de los datos agrupados mostró que el peso corporal aumentó 98 g por cada centímetro que aumentó la circunferencia de la pechuga. 
En la Figura 1 se muestra el ajuste lineal de la regresión del peso corporal sobre la circunferencia de la pechuga para datos de hembras, datos de machos y datos agrupados de gallinas de ambos sexos. Las ecuaciones de regresión estimadas fueron: para machos peso corporal $(\mathrm{kg})=-1.812+(0.1259 \mathrm{x}$ circunferencia de la pechuga $)$; para hembras peso corporal $(\mathrm{kg})=-0.3255+(0.061 \mathrm{x}$ circunferencia de la pechuga $)$ y para los datos agrupados peso corporal $(\mathrm{kg})=-1.274+(0.098 \times$ circunferencia de la pechuga $)$. Los errores estándar del intercepto y del coeficiente de regresión fueron: 0.405 y $0.013,0.226$ y 0.008, y 0.222 y $0.007 \mathrm{~kg}$, para datos de machos, datos de hembras y los datos agrupados, respectivamente.

Figura 1: Regresión lineal del peso corporal sobre la circunferencia de la pechuga de machos, hembras y machos y hembras agrupados.

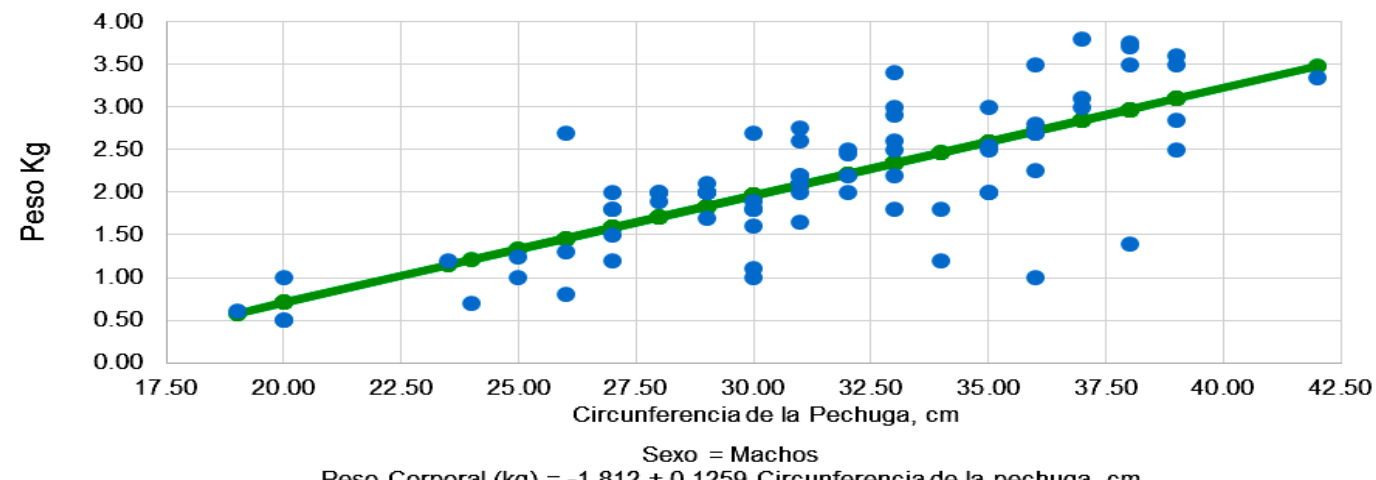

Peso Corporal $(\mathrm{kg})=-1.812+0.1259$ Circunferencia de la pechuga, $\mathrm{cm}$
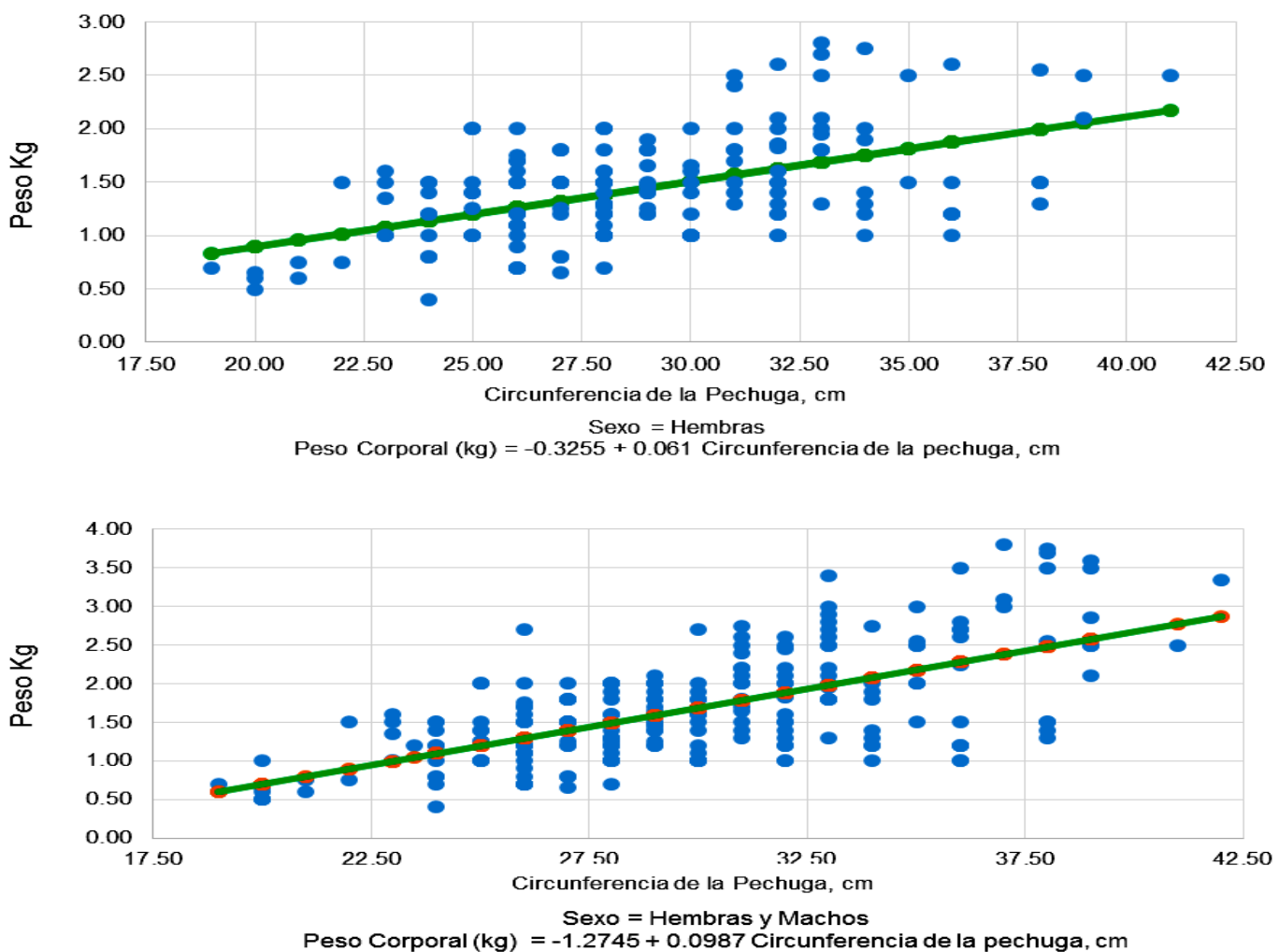
En el Cuadro 4 se muestran los coeficientes de correlación de Pearson estimados a partir de los datos individuales de machos y hembras, mientras que en el Cuadro 5 se presentan los coeficientes de correlación estimados a partir de los datos de machos y hembras agrupados. En todos los casos las correlaciones fueron de menor magnitud para hembras que para machos. En hembras la asociación más alta, aunque de magnitud moderada, se observó para peso corporal con circunferencia de la pechuga $(\mathrm{r}=0.52)$. Las correlaciones de envergadura con longitud del tarso y peso corporal fueron las más bajas ( $r=0.08$ y $r=0.13$, respectivamente); en contraste, éstas fueron moderadas en machos ( $r=0.55$ y $r=0.49$, respectivamente).

Cuadro 4: Coeficientes de correlación de Pearson para variables morfológicas cuantitativas de gallinas machos (debajo de la diagonal) y hembras (arriba de la diagonal)

\begin{tabular}{lllccc}
\hline Variable & LC & ENV & CP & LT & PC \\
\hline LC & & $0.35^{\mathrm{b}}$ & $0.20^{\mathrm{b}}$ & $0.17^{\mathrm{c}}$ & $0.20^{\mathrm{b}}$ \\
ENV & $0.46^{\mathrm{b}}$ & & $0.34 \mathrm{~b}$ & 0.08 & 0.13 \\
CP & $0.56^{\mathrm{b}}$ & $0.63^{\mathrm{b}}$ & & 0.13 & $0.52^{\mathrm{b}}$ \\
LT & $0.43^{\mathrm{b}}$ & $0.55^{\mathrm{b}}$ & $0.56^{\mathrm{b}}$ & & $0.41^{\mathrm{b}}$ \\
PC & $0.65^{\mathrm{b}}$ & $0.49^{\mathrm{b}}$ & $0.76^{\mathrm{b}}$ & $0.67^{\mathrm{b}}$ & \\
\hline
\end{tabular}

$\mathrm{LC}=$ longitud del cuerpo; $\mathrm{ENV}=$ envergadura; $\mathrm{CP}=$ circunferencia de la pechuga; $\mathrm{LT}=$ longitud del tarso; $\mathrm{PC}=$ peso corporal.

${ }^{\mathrm{b}}$ Coeficiente de correlación altamente significativo $(P<0.01)$.

${ }^{\mathrm{c}}$ Coeficiente de correlación significativo $(P<0.05)$.

Cuadro 5: Coeficientes de correlación de Pearson para variables morfológicas cuantitativas de gallinas de traspatio (datos de machos y hembras agrupados)

\begin{tabular}{lcccc}
\hline Variable & LC & ENV & CP & LT \\
\hline ENV & $0.58^{\mathrm{b}}$ & & & \\
CP & $0.60^{\mathrm{b}}$ & $0.67^{\mathrm{b}}$ & & \\
LT & $0.44^{\mathrm{b}}$ & $0.47^{\mathrm{b}}$ & $0.43^{\mathrm{b}}$ & \\
PC & $0.58^{\mathrm{b}}$ & $0.54^{\mathrm{b}}$ & $0.69^{\mathrm{b}}$ & $0.57^{\mathrm{b}}$ \\
\hline
\end{tabular}

$\mathrm{LC}=$ longitud del cuerpo; $\mathrm{ENV}=$ envergadura $\mathrm{CP}=$ circunferencia de la pechuga; $\mathrm{LT}=$ longitud del tarso; $\mathrm{PC}=$ peso corporal.

${ }^{\mathrm{b}}$ Coeficiente de correlación altamente significativo $(P<0.01)$. 
En machos el peso corporal tuvo asociaciones de moderadas a altas con longitud corporal $(\mathrm{r}=0.65)$, longitud del tarso $(\mathrm{r}=0.67)$ y circunferencia de la pechuga $(\mathrm{r}=0.76)$. Con la información agrupada de machos y hembras, todos los coeficientes de correlación fueron diferentes de cero $(P<0.0001)$, positivos y de moderados a altos, indicando el incremento de una variable al incrementarse las otras. Las correlaciones mayores se observaron para circunferencia de la pechuga con envergadura $(r=0.67)$, peso corporal $(r=0.69)$ y longitud del cuerpo $(r=0.60)$, y las más bajas para longitud del tarso con envergadura $(r=0.47)$, longitud del cuerpo $(\mathrm{r}=0.44)$ y circunferencia de la pechuga $(\mathrm{r}=0.43)$. Las correlaciones fenotípicas de peso corporal con longitud del cuerpo, envergadura y longitud del tarso fueron de magnitud moderada $(r=0.58, r=0.54$ y $r=0.57$, respectivamente).

En un estudio realizado para estimar parámetros genéticos y fenotípicos para peso corporal y medidas corporales lineales en líneas puras y cruzadas de gallinas indígenas de $\mathrm{Nigeria}^{(20)}$, reportaron correlaciones fenotípicas de peso corporal a las 20 semanas con longitud del cuerpo y longitud del tarso de 0.13 vs 0.57 , y 0.19 vs 0.57 , respectivamente. En ambos casos la magnitud de las correlaciones fue menor a las obtenidas en este estudio. Por otro lado, las correlaciones entre peso corporal con envergadura y circunferencia de la pechuga fueron similares en magnitud a las aquí obtenidas $\left(\begin{array}{llllllll}0.44 & 0.54 & 0.50 & \text { vs } & 0.69 \text {, }\end{array}\right.$ respectivamente). La correlación fenotípica entre circunferencia de la pechuga y envergadura fue de las más altas en ambos estudios (0.84 vs 0.67). En gallinas indígenas de áreas rurales de Nigeria ${ }^{(21)}$, se encontraron asociaciones entre peso a la 20 semanas con longitud del cuerpo y longitud del tarso de 0.58 y 0.57 , respectivamente. La correlación fenotípica entre longitud del cuerpo y longitud del tarso fue de 0.64, mayor a la obtenida en este estudio (0.44). En gallinas indígenas en Senegal ${ }^{(22)}$ se encontró que el peso corporal estuvo altamente asociado con la circunferencia de la pechuga (0.80) y medianamente con la longitud del cuerpo (0.68). Estos mismos autores reportaron que gallinas y gallos adultos aumentaron 74 y $38.8 \mathrm{~g}$, respectivamente, de peso corporal por cada centímetro que aumentó la circunferencia de la pechuga. La magnitud de las diferencias entre machos y hembras fue similar a la encontrada en este estudio.

Las variables estudiadas mostraron que las gallinas de traspatio en México presentan una diferenciación morfológica importante entre machos y hembras, excepto para robustez. Las correlaciones entre características, en todos los casos, fueron de menor magnitud para hembras que para machos, siendo de magnitud moderada a baja en hembras y moderada a alta en machos. 


\section{Agradecimientos}

Al INIFAP por el financiamiento del proyecto fiscal No. 10551832012, "Identificación de los recursos genéticos pecuarios para su evaluación, conservación y utilización sustentable en México. Aves y Cerdos", y al "Programa de Cooperación Científica y Tecnológica México-Italia 2014" por el apoyo para la realización del proyecto "La variabilidad genética en las razas avícolas autóctonas Italianas y Mexicanas: genética, el análisis filogenético y la interacción genotipo-ambiente".

\section{- Literatura citada}

1. Aquino RE, Arroyo LA, Torres HG, Riestra DD, Gallardo LF, López YB. El guajolote criollo (Meleagris gallopavo $L$.) y la ganadería familiar en la zona centro del estado de Veracruz. Téc Pecu Méx;2003;41(2):165-173.

2. Guevara HF, Ramírez DCA, Sanabria GN, Hernández LA, Gomez CH, Pinto RR, Medina JFJ. Gallinas de traspatio en la Frailesca, Chiapas: ¿Una alternativa en tiempos de incertidumbre? En Perezgrovas GR, et al editores, El traspatio iberoamericano. Experiencias y reflexiones en Argentina, Bolivia, Brasil, España, México y Uruguay. Talleres gráficos de la Universidad Autónoma de Chiapas. 2011.

3. Gutiérrez-Triay MA, Segura JC, López L, Santos J, Santos RH, Sarmiento L, Carvajal M, Molina G. Características de la avicultura de traspatio en el municipio de Tetiz, Yucatán, México. Trop Subtrop Agroecosyst 2007;7(3):217-224.

4. Zaragoza ML, Rodríguez HJV, Hernández ZJS, Perezgrovas GR, Martínez CB, Méndez EJA. Caracterización de gallinas Batsi alak en las tierras altas del sureste de México. Arch Zootec 2013;62(239):321-332.

5. Molina MP. Comparación de dos sistemas de producción y de manejo sanitario de las aves criollas de traspatio en los municipios de Ignacio de la Llave y Teocelo, Veracruz [tesis licenciatura]. Veracruz, México: Universidad Veracruzana; 2013.

6. Perezgrovas GR, Jerez SMP, Camacho EMA. Gallinas criollas y guajolotes nativos de México. Características y sistemas de producción. 1. ${ }^{a}$ ed. Chiapas: México; 2014. 
7. Sánchez-Sánchez M, Torres-Rivera JA. Diagnóstico y tipificación de unidades familiares con y sin gallinas de traspatio en una comunidad de Huatusco, Ver., México. Avances en Investigación Agropecuaria 2014;18(2):63-75.

8. Segura-Correa JC. Avicultura de traspatio y comportamiento productivo de la gallina criolla en Yucatán. En: Perezgrovas GR et al. editores. Características y sistemas de producción. 1. a ed. Universidad Autónoma de Chiapas. Tuxtla Gutiérrez, Chiapas, México; 2014:177-198.

9. FAO (Food and Agriculture Organization of the United Nations). Phenotypic characterization of animal genetic resources. FAO Animal Production and Health Guidelines No. 11. Rome. 2012.

10. SAS. Statistical Analysis System, User's Guide. Cary. NC, USA. SAS Inst. Inc. 2012.

11. Lázaro GC, Hernández ZJS, Vargas LS, Martínez LA, Pérez AR. Uso de caracteres morfométricos en la clasificación de gallinas locales. Actas Iberoamericanas de Conservación Animal 2012;2:109-114.

12. Olawunmi OO, Salako AE, Afuwape AA. Morphometric differentiation and assessment of function of the Fulani and Yoruba Ecotype indigenous chickens of Nigeria. Int J Morphol 2008;26(4):975-980.

13. Jerez-Salas MP, Vásquez-Dávila MA, Chávez-Cruz F, Pérez-León MI, CamachoEscobar MA, Carrillo-Rodríguez JC. Descriptores y variabilidad fenotípica de las gallinas criollas (Gallus gallus L.) en localidades de Oaxaca. En: Perezgrovas R., et al. editores. Características y sistemas de producción. 1. a ed. Chiapas, México: Universidad Autónoma de Chiapas; 2014:43-59.

14. Duran LM, Precucho GY. Caracterización fenotípica de las gallinas criollas de la provincia de Ocaña en los municipios de Cachira, Villacaro, La Esperanza, El Carmen y Gonzales [tesis licenciatura]. Colombia: Universidad Francisco de Paula Santander Ocaña; 2014.

15. Méndez TY. Zoometría comparada en las gallinas baleares [tesis maestría]. Córdoba, España: Universidad de Córdoba; 2010.

16. Francesch A, Villalba I, Cartañà M. Methodology for morphological characterization of chicken and its application to compare Penedesenca and Empordanesa breeds. Anim Genetic Resour 2011;48:79-84. 
17. Villacís RGE, Escudero SG, Cueva CF, Luzuriaga AR. Características fenotípicas de las gallinas criollas de comunidades rurales del sur del Ecuador. Centro de Biotecnología 2014; 3 (1):38-43. http://unl.edu.ec/sites/default/files/investigacion/ revistas/2014-12-1/bio_art4.pdf. Consultado 28 Abr, 2017.

18. Jiménez LM, Varón SA, Mendoza LF, Leal JD, Sánchez CA, Pinilla YC. Caracterización fenotípica de la gallina criolla de traspatio en tres regiones rurales de Colombia. Actas Iberoamericanas de Conservación Animal 2014;4 56-58.

19. Jáuregui R, Flores H, Vásquez L, Oliva MJ. Caracterización morfométrica de la gallina de cuello desnudo (Gallus domesticus nudicollis) en la región ch'ortí de Chiquimula, Guatemala. Ciencia, Tecnología y Salud 2015;2(1):2409-3459.

20. Adeleke MA, Peters SO, Ozoje MO, Ikeobi CON, Bamgbose AM, Adebambo OA. Genetic parameter estimates for body weight and linear body measurements in pure and crossbred progenies of Nigerian indigenous chickens. Lives Res Rural Develop 2011;23(1). http://www.lrrd.org/lrrd23/1/adel23019.htm. Consultado 28 Abr, 2017.

21. Ohagenyi IJ, Udokainyang AD, Ogbu KI, Obalisa A, Ukpe NE. Genetic correlations of some traits of the heavy ecotype of Nigerian native chicken. J Anim Sci Adv 2013:3(3):134-141.

22. Guèye EF, Ndiaye A, Branckaert RDS. Prediction of body weight on the basis of body measurements in mature indigenous chickens in Senegal. Lives Res Rural Develop 1998;(10). http://www.lrrd.org/lrrd10/3/sene103.htm. Consultado 20 Abr, 2017. 